\title{
Noise resistance territorial intensity-based optical flow using inverse confidential technique on bilateral function
}

\author{
Darun Kesrarat $^{1}$, Vorapoj Patanavijit ${ }^{2}$ \\ ${ }^{1}$ Department of Information Technology, Vincent Mary School of Science and Technology, Assumption University, \\ Bangkok, Thailand \\ ${ }^{2}$ Department of Computer and Network Engineering, Vincent Mary School of Engineering, Assumption University, \\ Bangkok, Thailand
}

\begin{tabular}{l} 
Article Info \\
\hline Article history: \\
Received May 26, 2021 \\
Revised Sep 7, 2021 \\
Accepted Oct 18, 2021 \\
\hline Keywords: \\
Bilateral filter \\
Error vector magnitude \\
Inverse confidential \\
Non-gaussian noise \\
Optical flow
\end{tabular}

\begin{abstract}
This paper presents the use of the inverse confidential technique on bilateral function with the territorial intensity-based optical flow to prove the effectiveness in noise resistance environment. In general, the image's motion vector is coded by the technique called optical flow where the sequences of the image are used to determine the motion vector. But, the accuracy rate of the motion vector is reduced when the source of image sequences is interfered by noises. This work proved that the inverse confidential technique on bilateral function can increase the percentage of accuracy in the motion vector determination by the territorial intensity-based optical flow under the noisy environment. We performed the testing with several kinds of non-Gaussian noises at several patterns of standard image sequences by analyzing the result of the motion vector in a form of the error vector magnitude (EVM) and compared it with several noise resistance techniques in territorial intensitybased optical flow method.
\end{abstract}

This is an open access article under the CC BY-SA license.

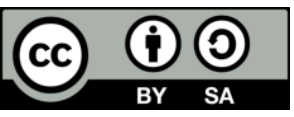

\section{Corresponding Author:}

Darun Kesrarat

Department of Information Technology

Vincent Mary School of Science and Technology, Assumption University

88 Moo 8 Bang Na-Trad Km. 26 Bangsaothong, Samuthprakarn 10570 Thailand

Email: darunksr@gmail.com

\section{INTRODUCTION}

The optical flow [1], [2] is a favorite specific method that it is used in numerous areas for more than 3 decades serving as movement prediction by pointing the motion vector (MV) as the main issue. In regular movement capture [3], [4], motion remuneration [5], image compression [6], and film encoding [7], [8], the MV is coded by using the optical flow method and it is applied in further processing. Also in image superresolution reconstruction areas [9], [10], the optical flow method is an essential part. At that point, the certainty result of optical flow is necessary. Basically, the optical flow is the coding method that it is used to point the MV base on the serial images. Numerous optical flow methods that were present like the global intensity-based method [11], section-based method [12], and phase-based method [13]. However, this paper attends to study the territorial intensity-based optical flow method [14]. T-OF is one of the accepted optical flow methods that using the intensity-based algorithm where it has been adjusted in numerous areas in early with a fine performance, coding speed, and noise resistance. Many research efforts may point to the improvement on coding speed [15], [16] but the efficiency of noise resistance is focused on in this paper as the main issue. 
In current situation, image and video transmission are a major application area of wireless communication systems. A peripheral noise reduces sensed image quality and affects look patterns. This effect of image noise interference on gaze distribution was mainly inclined by noise intensity rather than noise type. With the image noise interference, the coding of the optical flow is impacted directly and leads to the error in the result of MV. Many model emphasizes efficient transmission of images from one place to another with minimum possible errors [17]-[19]. Many solutions proposed the improvement in the quality of the interfered image or denoise process [20]-[22] in early-stage separately out of the optical flow. But these approaches took additional denoise processing. The other solution is the improvement on the optical flow coding itself for noise resistance by adding correlate function and algorithm in the optical flow coding like inverse confidential and bilateral function [23], [24]. And this is the main issue in this work where the effectiveness in noise resistance territorial intensity-based method optical flow was proved by using the technique of inverse confidential in accompany with bilateral function.

This paper focuses on the noise resistance territorial intensity-based optical flow method using the inverse confidential technique on bilateral function [25]. This paper intends to inspect the effectiveness of optical coding on non-Gaussian noises in contaminating with sequences image data set. In our experiment, the non-Gaussian noises like Satl\&Pepper at 2 levels of density, Speckle at 2 levels of variance, and Poisson are used in contaminating with the 4 patterns of standard image sequences like coastguard, foreman, akiyo, and container. In our effectiveness analysis, we used the error vector magnitude (EVM). The EVM regards the scale of fault in direction of the determined MV. The paper organization is regulated as follows. Part 2 describes the research methods in optical flow. Part 3 describes the measurement of the performance in noise resistance adjacent to the other noise resistance methods to confirm the satisfying result of T-RB in noise resistance. Part 4 describes the conclusion.

\section{RESEARCH METHODS IN TERRITORIAL INTENSITY-BASED METHOD OPTICAL FLOW AND NOISE RESISTANCE TECHNIQUES}

In this part, it is explained the territorial intensity-based optical flow method and the noise resistance techniques on this optical flow.

\subsection{Territorial intensity-based optical flow method}

The territorial intensity-based optical flow method (T-OF) [14] is an ordinary method for determining the MV in 2 dimension movement. The T-OF determine via the value of "intensity of luminous scale" and "smoothness deviation" [26], [27] with a weight least-square for each territorial. The formula is coded to determine the classical MV as:

$$
\left[\begin{array}{l}
u(x, y, k) \\
v(x, y, k)
\end{array}\right]=\left[\begin{array}{cc}
\sum I_{x}^{2}(x, y, k) & \sum I_{x}(x, y, k) I_{y}(x, y, k) \\
\sum I_{x}(x, y, k) I_{y}(x, y, k) & \sum I_{y}^{2}(x, y, k)
\end{array}\right]^{-1} \times\left[\begin{array}{l}
-\sum I_{x}(x, y, k) I_{k}(x, y, k) \\
-\sum I_{y}(x, y, k) I_{k}(x, y, k)
\end{array}\right]
$$

With the above formula for T-OF, the flow is constant in a territorial neary pixel. The formual for all the pixels in that territorial is solved by the least squares. A weight least-square is coded to determine the MV in each territorial. Where $I(x, y, k)$ indicate the intensity of luminous value at a position $(x, y)$ on an image at sequence no. $k$. in a territorial of vertical and horizontal alignment. The formula for intensity of luminous is:

$$
\begin{aligned}
& I x=1 / 4\{I x, y+1, k-I x, y, k+I x+1, y+1, k-I x+1, y, k+I x, y+1, k+1- \\
&I x, y, k+1+I x+1, y+1, k+1-I x+1, y, k+1\} \\
& I y /=1 / 4\{I x+1, y, k-I x, y, k+I x+1, y+1, k-I x, y+1, k+I x+1, y, k+1 \\
&-I x, y, k+1+I x+1, y+1, k+1-I x, y+1, k+1\} \\
& I t=1 / 4\{I x, y, k+1-I x, y, k+I x+1, y, k+1-I x+1, y, k+I x, y+1, k+1- \\
&I x, y+1, k+I x+1, y+1, k+1-I x+1, y+1, k\}
\end{aligned}
$$

\subsection{T-OF using bilateral function}

The bilateral function is one of the general famous noise removal functions. The use of the bilateral function is found to good effective result in noise resistance when it is coded in correlate with the optical flow called T-OF using the bilateral function (T-B) [23]. The main procedure is to code the bilateral function [28]-[31] over the determined MV from the T-OF. The bilateral formula over the determined MV from the T$\mathrm{OF}$ is:

$$
v_{b}(x)=\frac{1}{K} \sum_{|m|<M} v(x) \emptyset(x+m)
$$


With the above formula for T-B, the bilateral function is adopt to with averagely weighted from territorial MV. Where $K$ is the kernel normalization found on the conventional groundwork [28]. In our analysis, we referred territorial for $M$ by \pm 7 . The formula for kernel normalization $(K)$ is:

$$
K=\sum_{|m|<M} \emptyset(x+m)
$$

Where $m$ is the territorial group of bilateral gaussian kernel $(\phi)$. The formula for bilateral gaussian kernel $(\phi)$ is:

$$
\varnothing(x+n)=\exp \left(\frac{|n|^{2}}{2 \delta_{a}^{2}}+\frac{\left|I(x+n)-I(x)^{2}\right|}{2 \delta_{b}^{2}}\right)
$$

\subsection{T-OF using inverse confidential}

In the T-OF using inverse confidential (T-I) [24], the 2 ways hypothesis was proposed to identify confidence rate to ensure certainty of the MV from the optical flow. With the confidence function, the optical flow is coded to determine the MV in inverse direction and used to determine the confidential base on the following formula:

$$
C_{l}^{n}(s, k)=\exp \left(\frac{\left|v_{l}^{n}(s, k)+v_{l-}^{n}\left(s+v_{l}^{n}(s, k), k+1\right)\right|}{\frac{\left.\left|v_{l}^{n}(s, k)\right|+\left|v_{l-}^{n}\left(s+v_{l}^{n}(s, k), k+1\right)\right|\right)}{2+\beta}}\right)
$$

Where vl and vl- are ordinary MV and inverse MV, and $\mathrm{n}$ is the number of territorial MV. $\mathrm{s}$ is the point $(\mathrm{x}, \mathrm{y})$ on a 2-dimensional image and $\beta$ bypasses the division by nil in the formula. The confidence rates are used to code the ending MV in an average of territorial $(N(s O))$ by:

$$
\bar{v}_{l}^{n}\left(s_{0}\right)=\frac{\sum_{s_{i} \in N\left(s_{0}\right)} c_{l}^{n}\left(s_{i}\right) v_{l}^{n}\left(s_{i}\right)}{\sum_{s_{i} \in N\left(s_{0}\right)} c_{l}^{n}\left(s_{i}\right)}
$$

Where $\left(\bar{v}_{l}^{n}\left(s_{0}\right)\right)$ is coded from the confidence rate among territorial $N\left(s_{0}\right)$ based on the point $s_{0}=(x, y, k)$.

\subsection{T-OF using inverse confidential technique with bilateral function}

The T-OF using the inverse confidential technique with bilateral function (T-IB) [25] is the accompanying method between inverse confidential technique and bilateral function. The procedure of T-RB is illustrated in Figure 1.

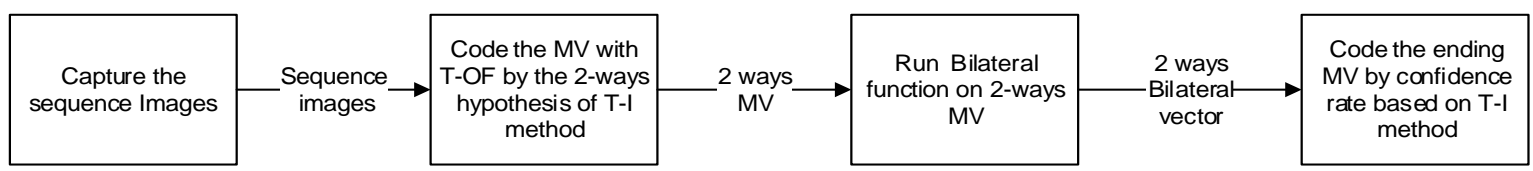

Figure 1. The procedure of T-IB

First, the traditional T-OF is used to code the MV under the 2 ways hypothesis in T-I. Consequently, the results of MV in 2 directions are used to calculate with bilateral function. After that, the determination $\mathrm{MV}$ is continued to process through a confidence rate based on the T-I method.

\section{EXPERIMENTAL AND CONSEQUENCE}

In our experimental, we focused mainly on T-OF and compared its performance against the other methods where the environment and configuration for the experimental was stated as follow. For T-OF, we adjusted the smoothness deviation with mask coefficient on 5 iteration loops of territorial $(5 \times 5)$ without pyramid for coding MV. 
About the standard image sequences, we adjusted 4 different patterns of QCIF $(176 \times 144)$ image sequences (coastguard, foreman, akiyo, and container) in line for 100 frames each. Figure 2 shows each illustration. And Poisson Noise, Speckle Noise at variance 0.01 and 0.05, and Salt\&Pepper Noise at density 0.005 and 0.025 are represented as the non-Gaussian noise used in the experiment. Entirely, there were 20 sets of the image sequence in the experiment along with the performance comparison of the proposed T-IB with classical T-OF, T-I, and T-B.

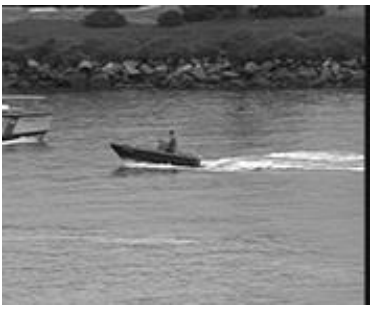

(a)

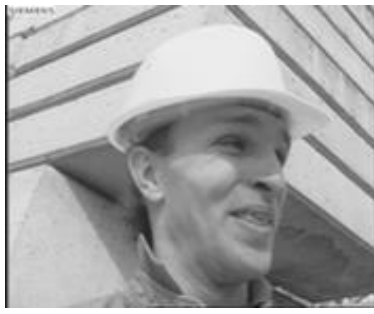

(b)

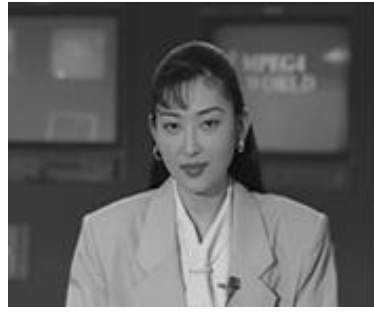

(c)

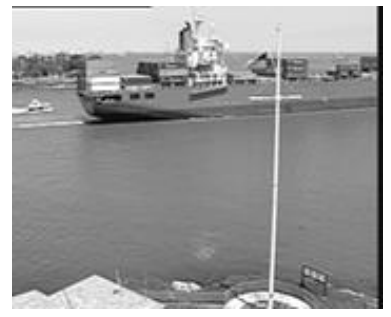

(d)

Figure 2. The image sequences on the experiment, (a) coastguard, (b) foreman, (c) akiyo, (d) container

Obviously, the consequence of noise resistance was determined by the EVM. The EVM is accurately to admeasure the MV for quantifying the performance. The low value in EVM means the improvement in noise resistance. Here, we adjusted only the active movement vector against the ground truth vector with root-mean-square by average.

Table 1 shows the compiled average EVM of each optical flow method through 100 frames on each kind of non-Gaussian noise in correspondence with each pattern of the standard image sequence. Figures 3 to Figure 6 present the "EVM frame outline" in comparison among several noise resistance optical flow methods over each kind of noise based on each pattern of standard image sequence by the graph.

Table 1. The compiled average EVM

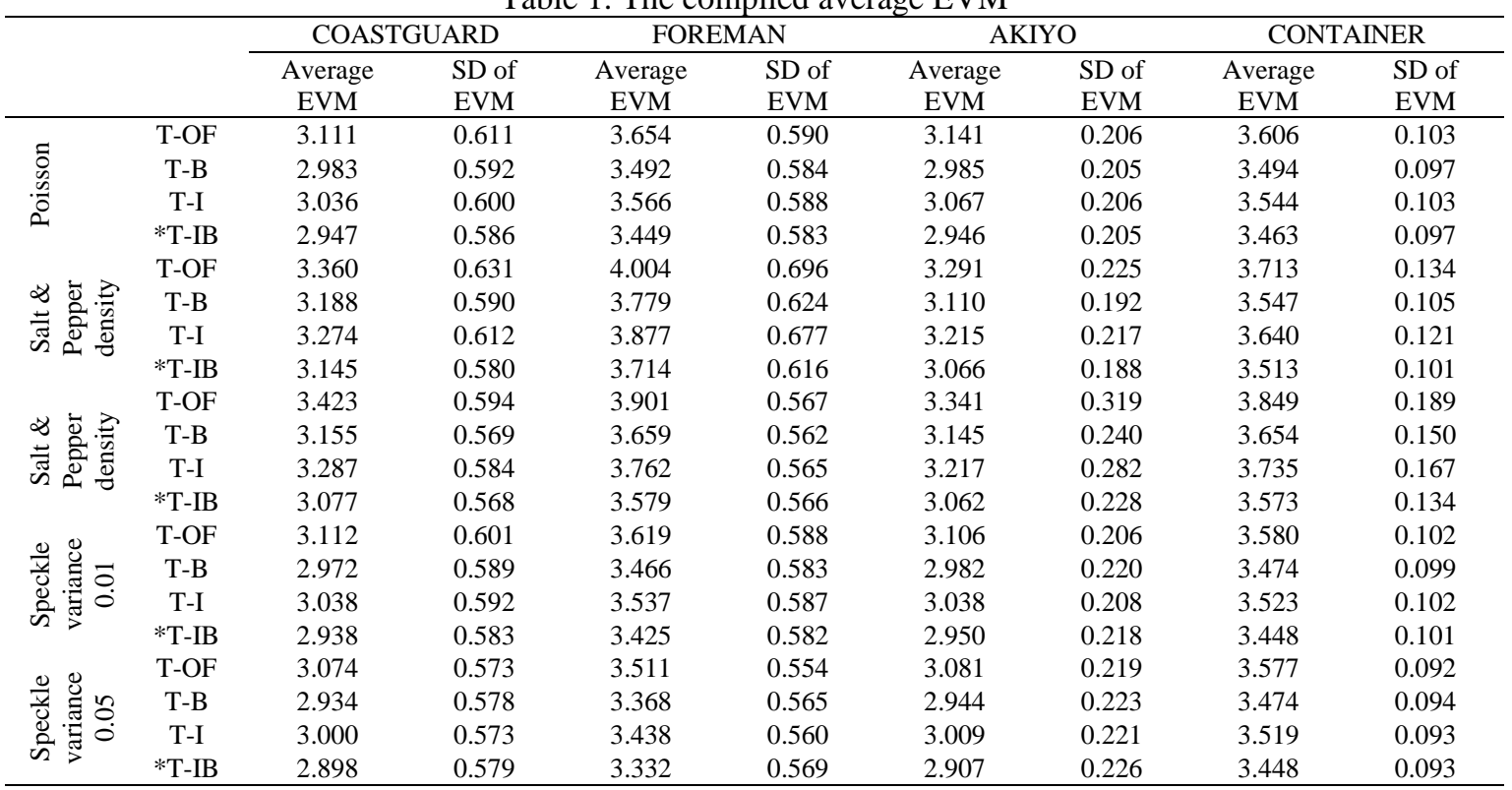




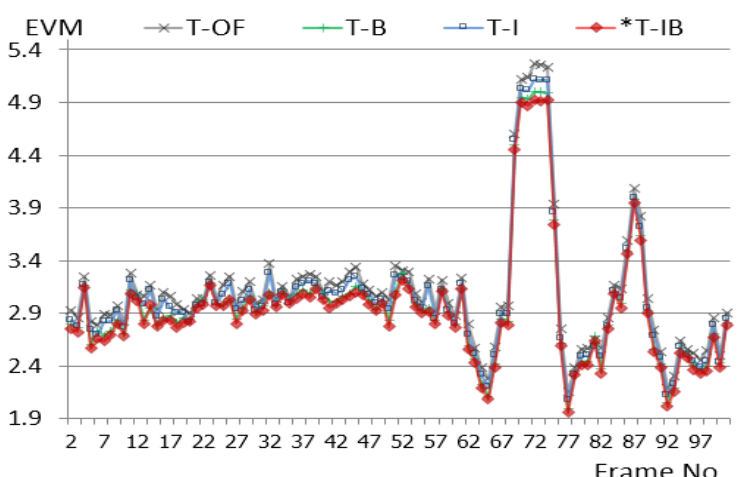

(a)

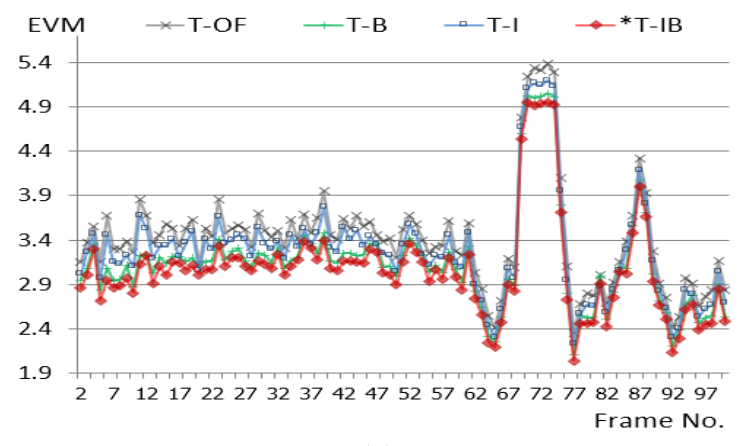

(c)

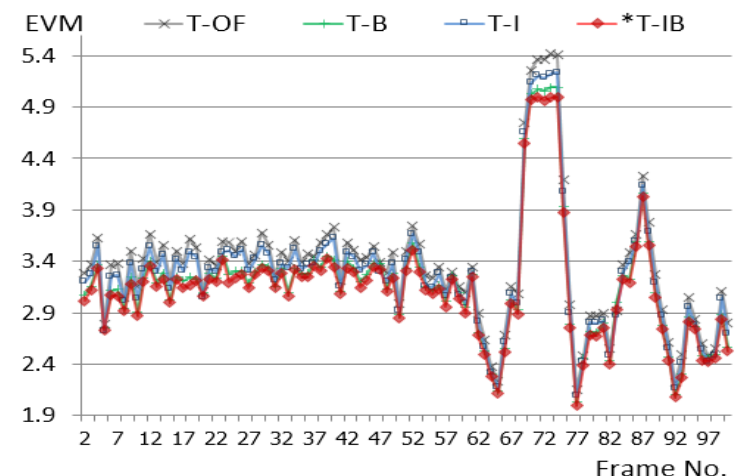

(b)

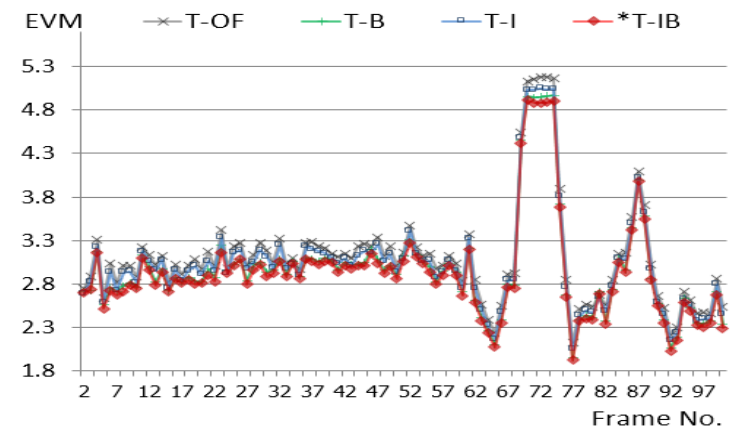

(d)

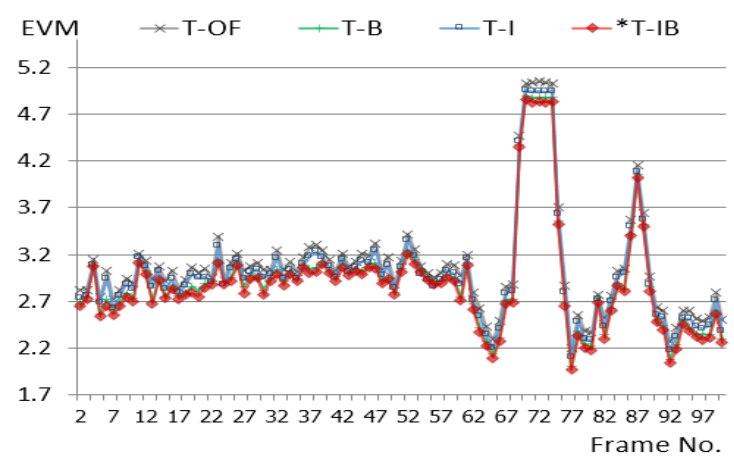

(e)

Figure 3. The EVM frame outline of COASTGUARD, (a) poisson, (b) salt \& pepper density 0.005 , (c) salt \& pepper density 0.025 ,(d) speckle variance 0.01 , (e) speckle variance 0.05

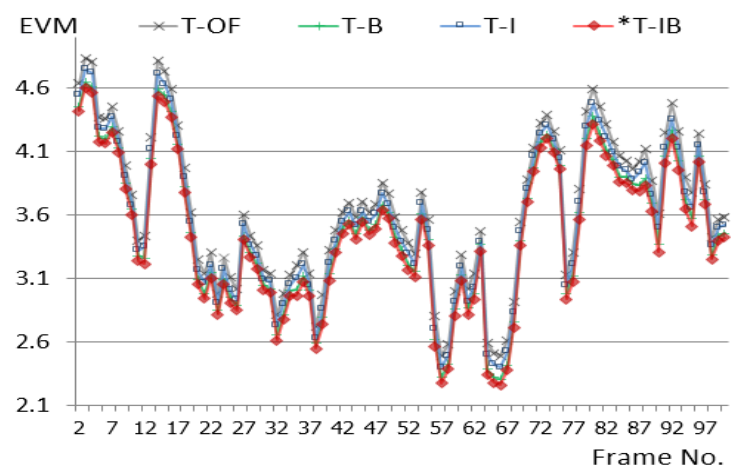

(a)

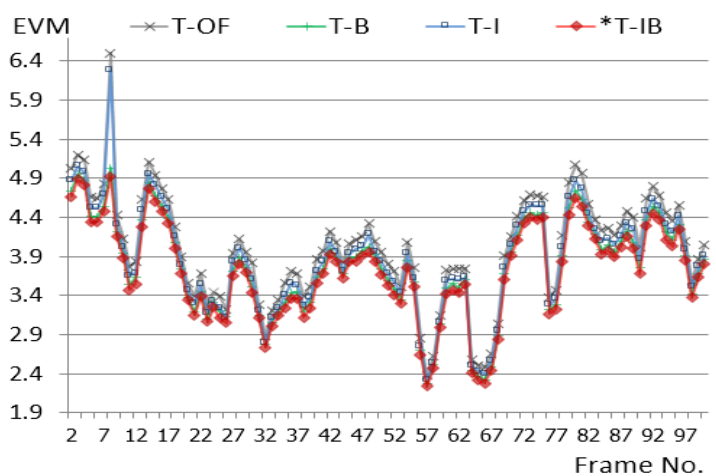

(b)

Figure 4. The EVM frame outline of FOREMAN, (a) poisson, (b) salt \& pepper density 0.005 


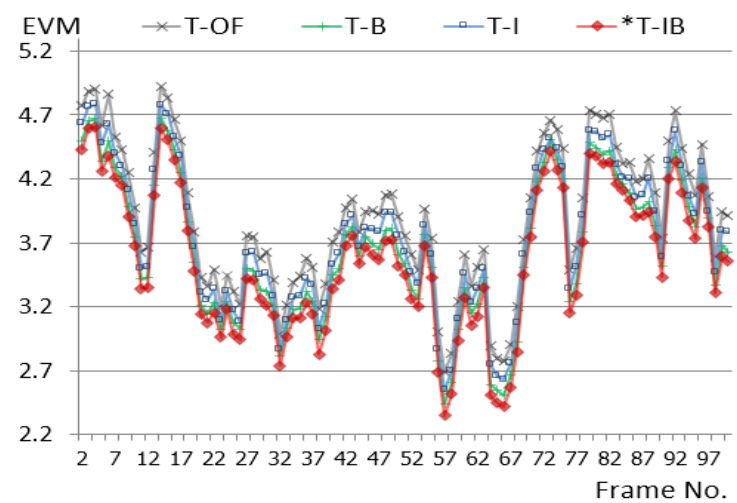

(c)

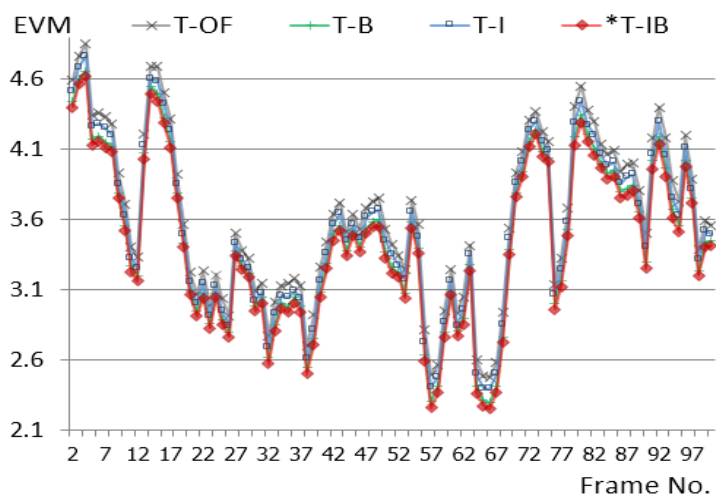

(d)

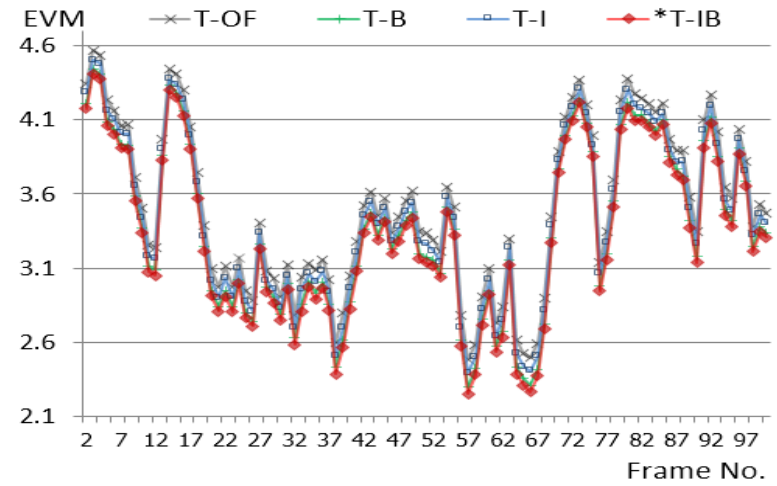

(e)

Figure 4. The EVM frame outline of FOREMAN, (c) salt \& pepper density 0.025 , (d) speckle variance 0.01 , (e) speckle variance 0.05 (continue)

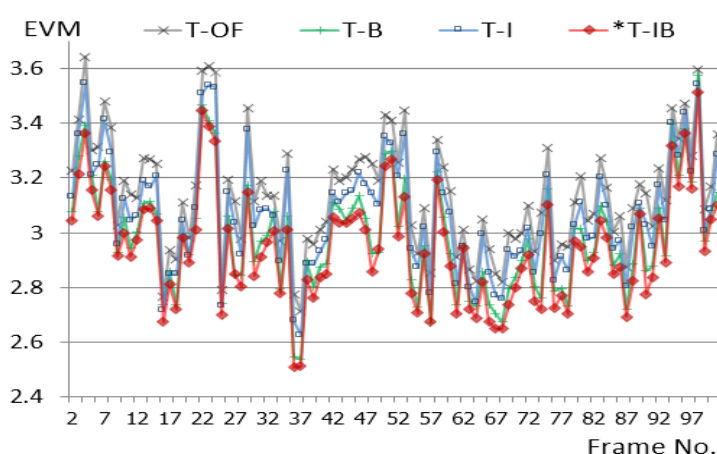

(a)

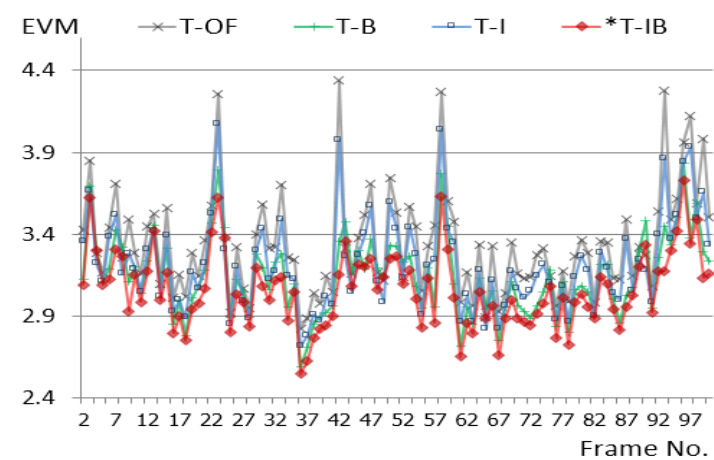

(c)

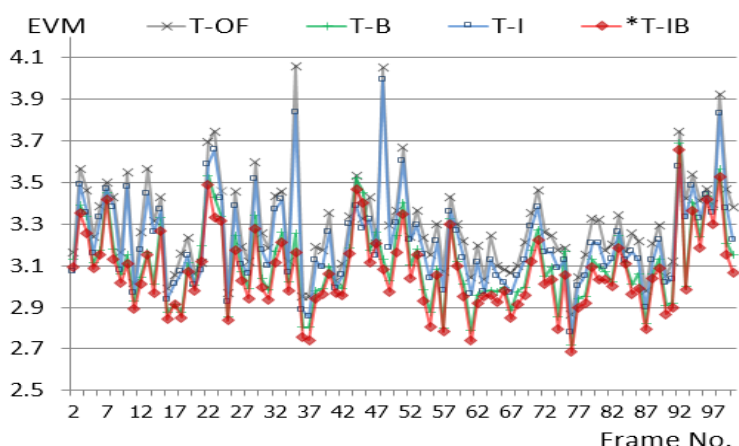

(b)

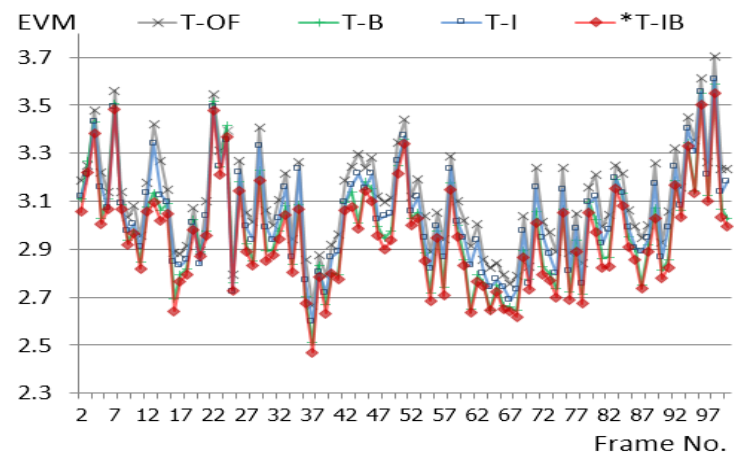

(d)

Figure 5. The EVM frame outline of AKIYO,(a) poisson, (b) salt \& pepper density 0.005, (c) salt \& pepper density 0.025 , (d) speckle variance 0.01 


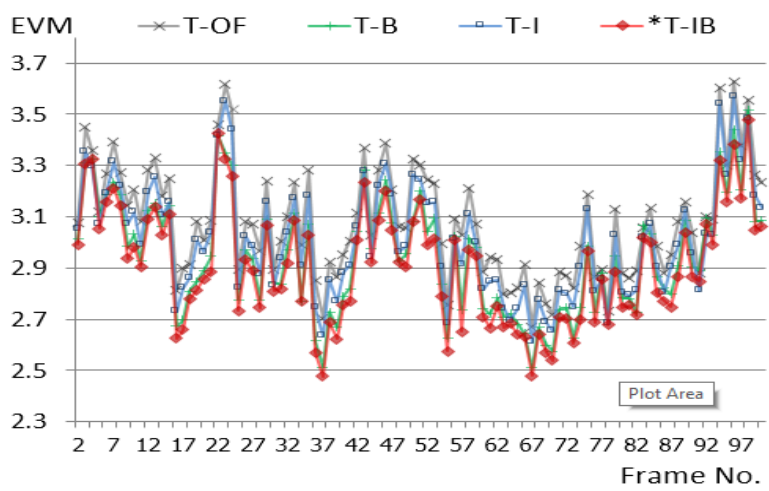

(e)

Figure 5. The EVM frame outline of AKIYO, (e) speckle variance 0.05 (continue)

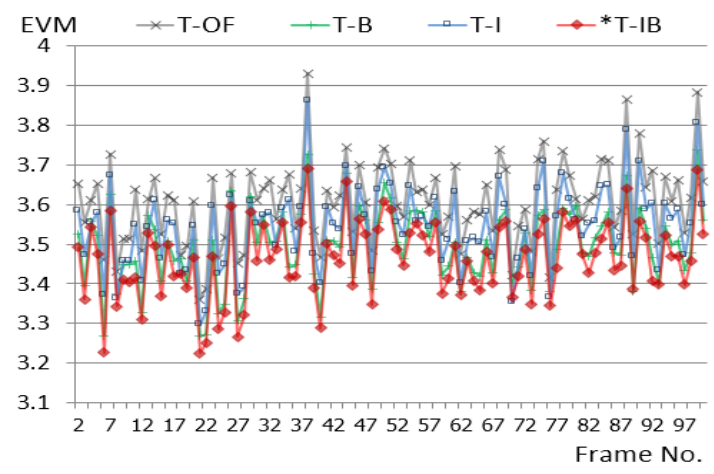

(a)

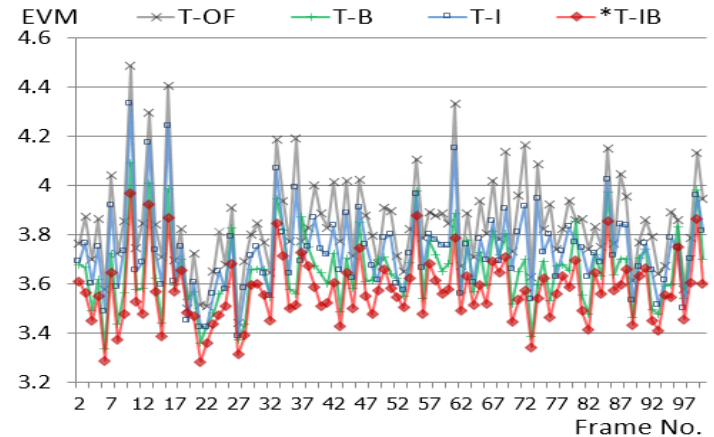

(c)

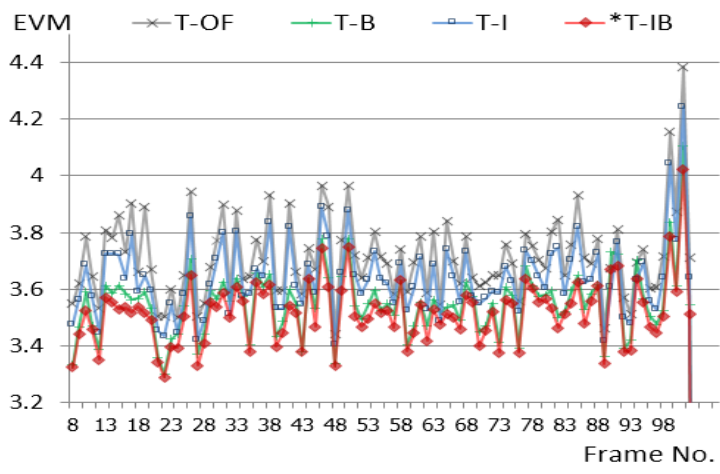

(b)

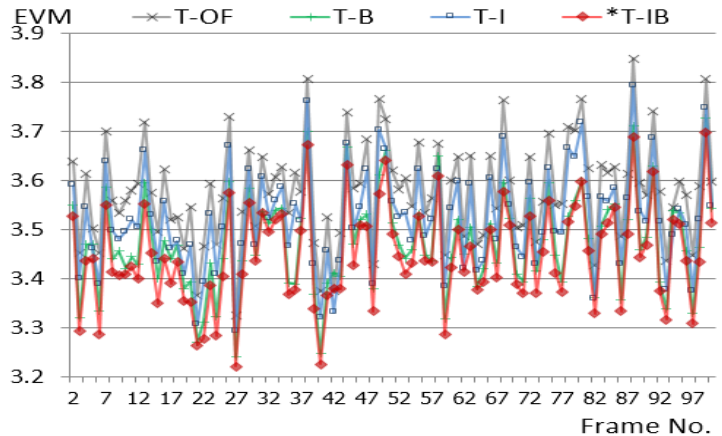

(d)

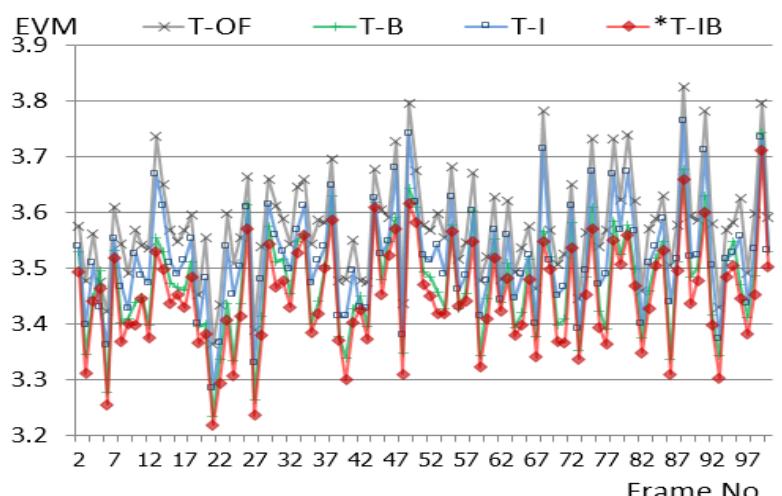

(e)

Figure 6. The EVM frame outline of CONTAINER,(a) poisson, (b) salt \& pepper density 0.005, (c) salt \& pepper density 0.025 , (d) speckle variance 0.01 , (e) speckle variance 0.05 


\section{CONCLUSION}

Regarding the character of territorial processing in T-OF, it provided a good result in noise resistance by traditional. Then, the overall result of noise resistance of T-I, T-B, and T-IB was slightly improved over T-OF. In our investigation, we proved that the proposed method (T-IB) presented the best noise resistance on the non-Gaussian noise overall. Then, the software areas such namely move to track within the noisy surroundings are appropriate in accordance with adopting this method in accordance with gain accuracy among the territorial intensity-based optical flow method. By the way, there is no beyond compare method at this moment. Regarding the detail in the frame outline, the performance of T-I, and T-B still presented the best result in noise resistance in a few frames. Then, the research on numerous kinds of noise with numerous noise resistance methods is a controversy for the prospective research.

\section{ACKNOWLEDGEMENTS}

This research project was funded by Assumption University of Thailand.

\section{REFERENCES}

[1] Andrew and J. R. Burton, "Thinking in Perspective: Critical Essays in the Study of Thought Processes," Routledge, ISBN 978-0-416-85840-2, 1978.

[2] D. H. Warren and E. R. Strelow, "Electronic Spatial Sensing for the Blind: Contributions from Perception," Springer, 1985.

[3] C. S. Royden and K. D. Moore, "Use of speed cues in the detection of moving objects by moving observers," Vision Research, vol. 59, pp. 17-24, 2012, doi: 10.1016/j.visres.2012.02.006.

[4] S. A. Mahmoudi, M Kierzynka, P. Manneback and K. Kurowski, "Real-time motion tracking using optical flow on multiple GPUs," Bulletin of the Polish Academy of Sciences. Technical Sciences, vol. 62, no. 1, pp. 139-150, 2014.

[5] Z. Chen, T. He, X. Jin and F. Wu, "Learning for Video Compression," IEEE Transactions on Circuits and Systems for Video Technology, vol. 30, no. 2, pp. 566-576, 2020, doi: 10.1109/TCSVT.2019.2892608.

[6] A. J. Qasim, R. Din and F. Q. A. Alyousuf, "Review on techniques and file formats of image compression," Bulletin of Electrical Engineering and Informatics, vol. 9, no. 2, pp. 602-610, 2020, doi: 10.11591/eei.v9i2.2085.

[7] T. Wiegand, G. J. Sullivan, G. Bjontegaard and A. Luthra, "Overview of the H.264/AVC video coding standard," in IEEE Transactions on Circuits and Systems for Video Technology, vol. 13, no. 7, pp. 560-576, July 2003, doi: 10.1109/TCSVT.2003.815165

[8] ISO, "Information Technology-Coding of Audio Visual Objects-Part 2: Visual, JTC1/SC29/WG11, ISO/IEC 14 469-2 (MPEG-4 Visual)," 2000, [Online]. Available: https://www.iso.org/standard/39259.html.

[9] C. Deng, J. Liu, W. Tian, S. Wang, H. Zhu and S. Zhang, "Image Super-Resolution Reconstruction Based On L1/2 Sparsity," Bulletin of Electrical Engineering and Informatics, vol. 3, no. 3, pp. 155-160, 2014.

[10] D. Kesrarat, K. Thakulsukant and V. Patanavijit, "A Novel Elementary Spatial Expanding Scheme Form on SISR Method with Modifying Geman \& Mcclure Function," Telecommunication, Computing, Electronics and Control TELKOMNIKA, vol. 17, no. 5, pp. 2554-2560, doi: 10.12928/TELKOMNIKA.v17i5.12799.

[11] K. P. Berthold and B. G. Schurnck, "Determining Optical Flow," Artificial Intelligence, vol. 17, pp. 185-203, 1981.

[12] T. R. Reed, "Digital Image Sequence Processing, Compression, and Analysis," Chemical Rubber Company (CRC) Press, 2004, doi: 10.1201/9780203486788.

[13] D. J. Fleet and A. D. Jepson, "Computation of component image velocity from local phase information " Computer Vision, vol. 5, pp.77-104, 1990.

[14] B. D. Lucas and T. Kanade, "An Iterative Image Registration Technique with an Application to Stereo Vision," Proceeding of Defense Advanced Research Projects Agency (DARPA) Image Understanding Workshop, pp. 121130, 1981.

[15] Y. Su, Y. Huang and C. J. Kuo, "Efficient Text Classification Using Tree-structured Multi-linear Principal Component Analysis," 24th International Conference on Pattern Recognition ICPR, 2018, pp. 585-590, doi: 10.1109/ICPR.2018.8545832.

[16] Y. Su, R. Lin and C. C. J. Kuo, "Tree-structured multi-stage principal component analysis (TMPCA): Theory and applications," Expert Systems with Applications, vol. 118, pp. 355-364, 2019, doi: 10.1016/j.eswa.2018.10.020.

[17] M. Kaur, L. Kansal, N. Kaur, G. S. Gaba and D. P. Agrawal, "Analysis of Image Transmission using MIMOAlamouti Space-Time Encoding," International Journal on Advanced Science, Engineering and Information Technology, vol. 9, no. 1, 2019.

[18] M. Kaur, L. Kansal and N. Kaur, "Image transmission using MIMO-OSTBC3 space-time encoding," Far East Journal of Electronics and Communications, vol. 17, no. 6, pp. 1437-1450, 2017, doi: 10.17654/EC017061437.

[19] L. Kansal, G. S. Gaga, N. Chilamkurti and B. G. Kim, "Efficient and Robust Image Communication Techniques for 5G Applications in Smart Cities," Energies, vol. 14, no. 13, 2021, doi: 10.3390/en14133986.

[20] V. Patanavijit and K. Thakulsukanant, "A Computational Experimental Investigation of Noise Suppressing Technique Stand on Hard Decision Threshold Dissimilarity under Fixed-Intensity Impulse Noise," Indonesian Journal of Electrical Engineering and Computer Science, vol. 24, no. 1, pp. 144-156, 2021, doi: 10.11591/ijeecs.v24.i1.pp144-156. 
[21] V. Patanavijit and K. Thakulsukanant, "The Novel Noise Classification Techniques Found on QTSD (Quadruple Threshold Statistical Detection) Filter under FIIN Environment," Bulletin of Electrical Engineering and Informatics, vol. 10, no. 5, pp. 2520-2529, 2021, doi: 10.11591/eei.v10i5.3105.

[22] L. Abderrahim, M. Salama and D. Abdelbaki, "Novel design of a fractional wavelet and its application to image denoising," Bulletin of Electrical Engineering and Informatics, vol. 9, no. 1, pp. 129-140, 2020, doi: 10.11591/eei.v9i1.1548.

[23] D. Kesrarat and V. Patanavijit, "Experimental Analysis of Performance Comparison on Both Linear Filter and Bidirectional Confidential Technique for Spatial Domain Optical Flow Algorithm," The Electrical Engineering/Electronics, Computer, Communications and Information Technology Association Transactions on Computer and Information Technology, vol. 7, no. 2, pp. 157-168, 2013.

[24] R. Li and S. Yu, "Confidence based optical flow algorithm for high reliability," IEEE International Conference on Acoustics, Speech and Signal Processing, 2008, pp. 785-788, doi: 10.1109/ICASSP.2008.4517727.

[25] D. Kesrarat and V. Patanavijit, "Bidirectional confidential with bilateral filter on local based optical flow for image reconstruction under noisy condition," IEEE/ACIS 16th International Conference on Software Engineering, Artificial Intelligence, Networking and Parallel/Distributed Computing (SNPD), 2015, pp. 1-4, doi: 10.1109/SNPD.2015.7176211.

[26] J. L. Barron, D. J. Fleet and S. S. Beauchemin, "Performance of Optical Flow Techniques," International Journal of Computer Vision, vol. 12, pp. 43-77, 1994.

[27] D. Fleet and Y. Weiss, "Optical Flow Estimation," Handbook of Mathematical Models in Computer Vision, Springer, pp. 237-257, 2006.

[28] S. Paris, P. Konprobst, J. Tumblin, F. Durand, "Bilateral filtering: Theory and Applications," Found. Trends Compt. Graph. Vis., vol. 4, no. 1, pp.1-73, 2008.

[29] M. G. Mozerov, "Constrained Optical Flow Estimation as a Matching Problem," IEEE Transactions on Image Processing, vol. 22, no. 5, pp. 2044-2055, 2013, doi: 10.1109/TIP.2013.2244221.

[30] Jian Sun, Yin Li, S. B. Kang and Heung-Yeung Shum, "Symmetric stereo matching for occlusion handling," IEEE Computer Society Conference on Computer Vision and Pattern Recognition (CVPR'05), 2005, pp. 399-406 vol. 2, doi: 10.1109/CVPR.2005.337.

[31] Ming Zhang and Bahadir Gunturk, "A new image denoising method based on the bilateral filter," IEEE International Conference on Acoustics, Speech and Signal Processing, 2008, pp. 929-932, doi: 10.1109/ICASSP.2008.4517763.

\section{BIOGRAPHIES OF AUTHORS}

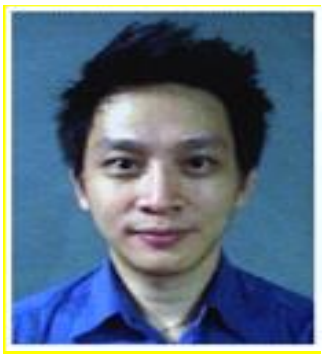

Darun Kesrarat received the B. S., M. S., and Ph. D. from the Department of Information Technology at Assumption University, Bangkok, Thailand. He is currently an Assistance Professor. His research areas include signal processing on Image/Video Reconstruction, SRR (Super-Resolution Reconstruction), Motion Estimation, and Optical Flow Estimation.

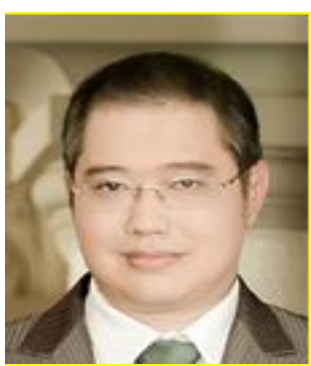

Vorapoj Patanavijit received the B.Eng., M.Eng., and Ph.D. degrees from the Department of Electrical Engineering at the Chulalongkorn University, Bangkok, Thailand, in 1994, 1997 and 2007 respectively. He is currently an Associate Professor. He works in the field of signal processing and multidimensional signal processing, specializing, in particular, on Image/Video Reconstruction, SRR (Super-Resolution Reconstruction), Compressive Sensing, Enhancement, Fusion, Digital Filtering, Denoising, Inverse Problems, Motion Estimation, Optical Flow Estimation and Registration. 